\title{
The Peroxisomal Targeting Signal 1 in sterol carrier protein 2 is autonomous and essential for receptor recognition
}

\author{
Chris P Williams ${ }^{1,2}$, Nicole Schueller ${ }^{1}$, Colin A Thompson ${ }^{3}$, Marlene van den Berg' ${ }^{2}$, Simon D Van Haren², \\ Ralf Erdmann ${ }^{4}$, Charles S Bond ${ }^{5}$, Ben Distel ${ }^{2}$, Wolfgang Schliebs ${ }^{4}$, Matthias Wilmanns ${ }^{1}$, Will A Stanley ${ }^{1,6^{*}}$
}

\begin{abstract}
Background: The majority of peroxisomal matrix proteins destined for translocation into the peroxisomal lumen are recognised via a C-terminal Peroxisomal Target Signal type 1 by the cycling receptor Pex $5 \mathrm{p}$. The only structure to date of Pex $5 p$ in complex with a cargo protein is that of the C-terminal cargo-binding domain of the receptor with sterol carrier protein 2, a small, model peroxisomal protein. In this study, we have tested the contribution of a second, ancillary receptor-cargo binding site, which was found in addition to the characterised Peroxisomal Target Signal type 1.

Results: To investigate the function of this secondary interface we have mutated two key residues from the ancillary binding site and analyzed the level of binding first by a yeast-two-hybrid assay, followed by quantitative measurement of the binding affinity and kinetics of purified protein components and finally, by in vivo measurements, to determine translocation capability. While a moderate but significant reduction of the interaction was found in binding assays, we were not able to measure any significant defects in vivo.

Conclusions: Our data therefore suggest that at least in the case of sterol carrier protein 2 the contribution of the second binding site is not essential for peroxisomal import. At this stage, however, we cannot rule out that other cargo proteins may require this ancillary binding site.
\end{abstract}

\section{Background}

Pex5p, the major import receptor for peroxisomal matrix proteins, is known to carry folded proteins across the peroxisomal membrane by a signal assembled shuttling mechanism [1,2]. It has been found to recognise the type 1 Peroxisomal Targeting Signal (PTS1) - a C-terminal tripeptide of consensus sequence -[S/A/C]-[K/H/R]-[L/M]$\mathrm{CO}_{2}{ }^{-}$, carried by some 40 human proteins destined for the peroxisomal lumen. The PTS1 sequence binds to Pex5p in an extended conformation, which is accommodated in a deep cavity in the tetratricopeptide repeat (TPR) domain of Pex5p [3,4] (Figure 1A). An additional interface, remote from the PTS1 binding site, of about $500 \AA^{2}$ has been found to form between Pex5p and the model cargo protein sterol carrier protein 2 (SCP2),

\footnotetext{
* Correspondence: will.stanley@uwa.edu.au

'EMBL-Hamburg, c/o DESY, Notkestraße 85, 22603 Hamburg, Germany

Full list of author information is available at the end of the article
}

as demonstrated by the crystal structure and solution studies of SCP2 in complex with the C-terminal region of Pex5p [4]. In the following we will refer to the interface as "ancillary" or "secondary". This interface utilises the first and fourth $\alpha$-helices of SCP2 and helices 15 and 16 from the Pex5p C-terminal helical bundle (Figure 1A \&1B) and its formation confers an increased binding affinity of around 6-fold when compared to a minimal PTS1 hexapeptide (PGNAKL- $\mathrm{CO}_{2}{ }^{-}$), derived from the C-terminus of SCP2 [4]. Taken together, these observations may imply the presence of an additional receptor recognition element in SCP2, supplemental to the PTS1.

Inspection of the secondary interface of the $\operatorname{Pex} 5 \mathrm{p}(\mathrm{C})$ SCP2 complex structure reveals several intermolecular hydrogen bonds (Figure 1C), in particular: the carboxylate group of Glu35 from SCP2 interacts with the side chains of two Pex5p residues, Arg608 and Tyr620; Lys38 from SCP2 forms a salt bridge with Asp624 from Pex5p; finally, Lys28 from SCP2 interacts with the main chain carbonyl group
C Biomed Central

() 2011 Williams et al; licensee BioMed Central Ltd. This is an Open Access article distributed under the terms of the Creative Commons Attribution License (http://creativecommons.org/licenses/by/2.0), which permits unrestricted use, distribution, and reproduction in any medium, provided the original work is properly cited. 


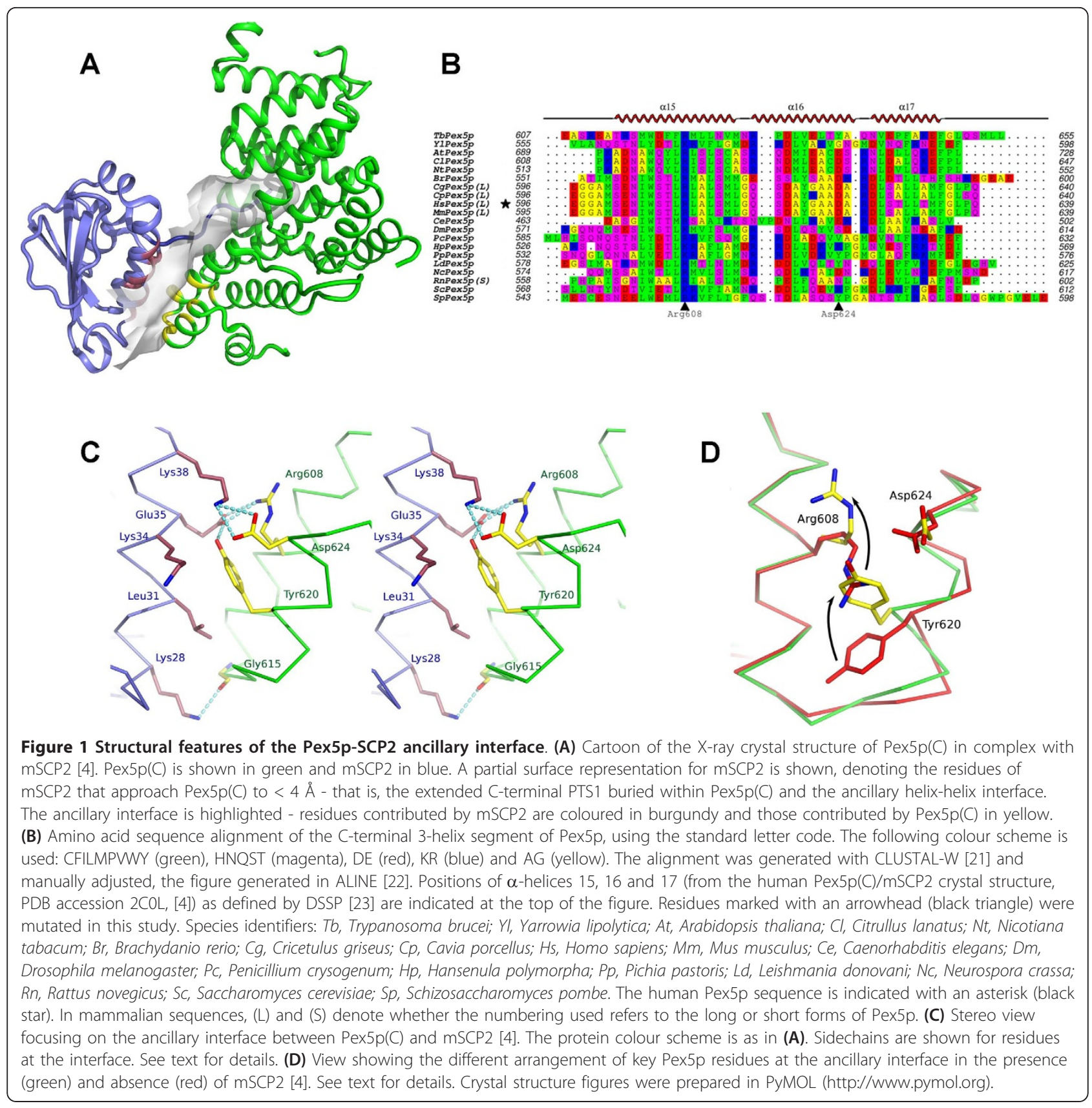

of Gly615 from Pex5p(C). Comparison with the Pex5p(C) apo-structure [4] reveals that in the absence of SCP2, two residues from this interface, Arg608 and Asp624, re-orient their sidechains to form an intramolecular salt bridge, inducing a re-orientation of the sidechain of Tyr620 (Figure 1D). An alignment of a set of Pex5p sequences (Figure 1B) indicates that while Tyr620 and Asp624 are only partly conserved, a basic residue at the position equivalent to Arg608 in human Pex5p is invariant.

In this study, we have made point mutations of two key residues of the ancillary SCP2 binding interface in Pex5p, Arg608 and Asp624, to tryptophans to assess the relative importance of the additional interface in the recognition of SCP2 by Pex5p. To dissect the contributions of the PTS1 and these additional interactions, we also used a version of SCP2 lacking the C-terminal PTS1, for comparison. By four different approaches we demonstrate that while receptor-cargo binding and SCP2 transfer into the peroxisome are absolutely dependent on the presence of the PTS1, the ancillary interface is found to be dispensable - mutations effecting binding do not inhibit peroxisomal import in vivo. These results indicate that the ancillary interface is unlikely to play an important role in the import of the PTS1 protein SCP2. 


\section{Results}

Yeast two-hybrid analysis of human Pex5p interactions with SCP2

First, we created mutant versions of the cargo-binding domain of Pex5p (Pex5p(C), consisting of residues 315-639) and tested their capacity to interact with different versions of SCP2 in the yeast two-hybrid system (Figure 2). As a control, we showed that the presence of the PTS1 of SCP2 is a pre-requisite for binding to $\operatorname{Pex} 5 \mathrm{p}(\mathrm{C})$, as no interaction could be detected in its absence, thus confirming previous findings [4]. The introduction of the mutation R608W into Pex5p $(C)$ did result in a three-fold reduction of the interaction with both pre- and $\mathrm{mSCP} 2$. In contrast, the $\operatorname{Pex} 5 \mathrm{p}(\mathrm{C})$ D624W mutation showed little affect, either leading to a slight decrease (mSCP2) or increase (preSCP2) of the interaction. To confirm that the observed decrease with the Pex5p(C) R608W construct was not due to problems with expression or stability of the protein, we performed Western blotting analysis with Pex5p antibodies on yeast lysates from cells expressing the different Pex5p(C) variants. We could see that all Pex5p constructs were expressed at similar levels (Figure 3).

\section{In vitro Pex5p-SCP2 binding}

We tested the capacity of these mutants to bind mSCP2 in vitro using isothermal titration microcalorimetry (ITC) (Table 1). We observed that the Pex5p(C) R608W mutation resulted in a two fold reduction in SCP2 binding, in agreement with our yeast-two-hybrid data. In addition, we found the binding stoichiometry reduced to 0.61 , indicating that only about two-thirds of the

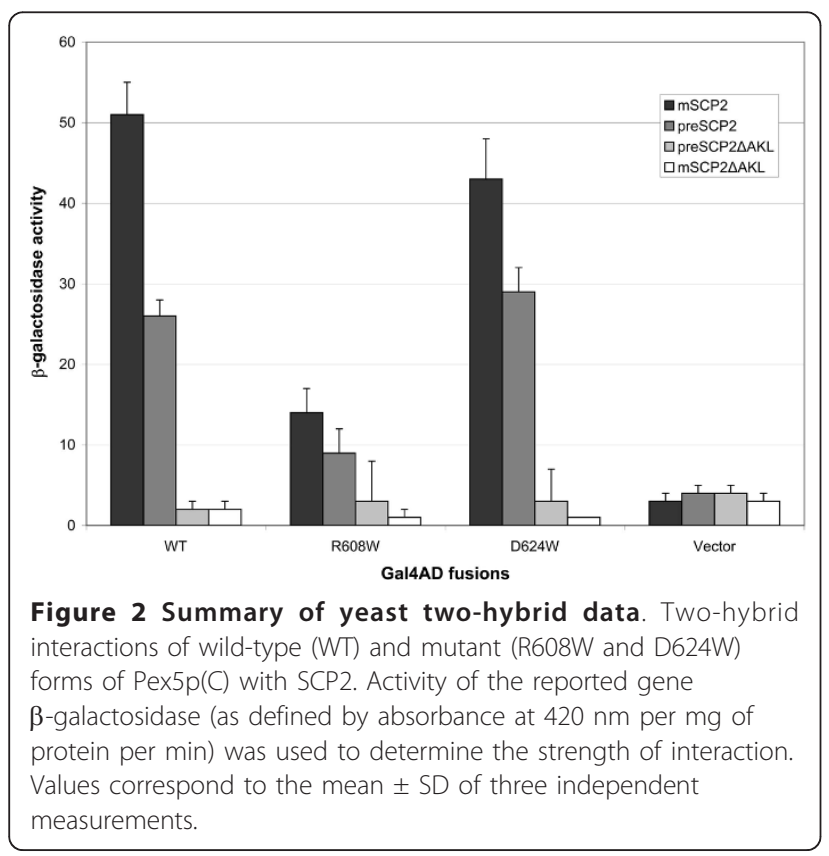

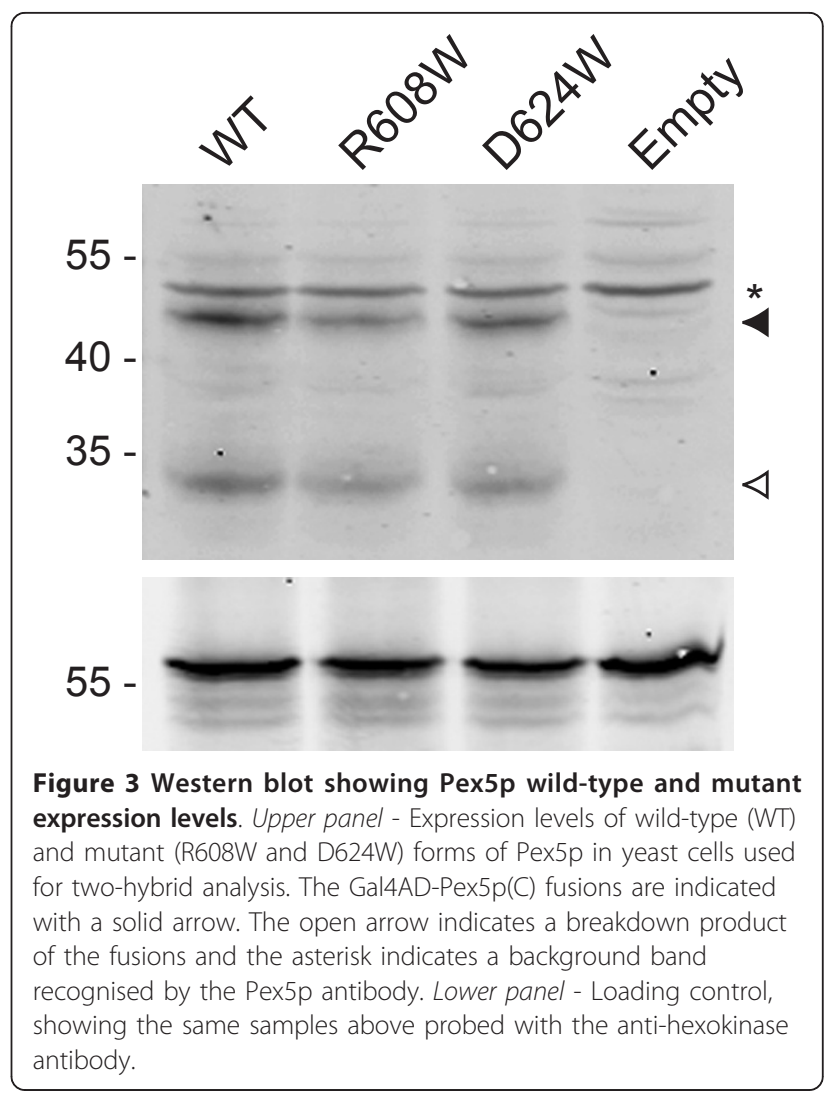

receptor was competent for SCP2 cargo binding, compared to a value approaching one for wild-type. The binding stoichiometry was further lowered to 0.41 when using the second $\operatorname{Pex} 5 \mathrm{p}(\mathrm{C})$ mutant, D624W, coupled with an apparent tighter binding when compared to wild-type $\operatorname{Pex} 5 \mathrm{p}(\mathrm{C})$. Additionally, we measured the binding affinity of the $\operatorname{Pex} 5 \mathrm{p}(\mathrm{C})$ mutants against a peptide derived from the C-terminus of SCP2 (Table 1, PGNAKL [4]), a binding event that is independent of the ancillary interface. The $\operatorname{Pex} 5 \mathrm{p}(\mathrm{C}) \mathrm{R} 608 \mathrm{~W}$ mutant bound to the peptide with a similar affinity as the wildtype protein. Furthermore, the binding stoichiometry of this mutant was closer to that of wild-type $\operatorname{Pex} 5 \mathrm{p}(\mathrm{C})$, suggesting that the R608W mutant is impaired in binding when utilising the secondary interface but not when using the PTS1 binding site alone.

To determine whether structural alterations induced by the mutations were the cause of the observed binding defects, we subjected the mutants to circular dichroism spectropolarimetry (CD) and static light scattering (SLS) analysis. While both mutants showed similar properties when tested with SLS (Table 2) (i.e. they are found to be monomeric and similarly mondispersed), a difference could be seen with the D624W mutant using CD (Figure 4, Table 3). Wild-type $\operatorname{Pex} 5 \mathrm{p}(\mathrm{C})$ is estimated to have $60.3 \pm 2.1 \% \alpha$-helical content and R608W $57.0 \pm$ 
Table 1 Summary of ITC data

\begin{tabular}{llccccc}
\hline Pex5p(C): & Ligand & $\mathbf{n}$ & $\begin{array}{c}\Delta \mathbf{H} \\
(\mathbf{k J / m o l})\end{array}$ & $\begin{array}{c}\mathbf{T} \Delta \mathbf{S} \\
(\mathbf{k J} / \mathbf{m o l})\end{array}$ & $\begin{array}{c}\Delta \mathbf{G} \\
(\mathbf{k J} / \mathbf{m o l})\end{array}$ & $\begin{array}{c}\mathbf{K}_{\mathbf{d}} \\
(\mathbf{n M})\end{array}$ \\
\hline Wild-type (5) ${ }^{\text {a }}$ & mSCP2 & $0.93 \pm 0.03$ & -43.4 & -1.8 & -42.3 & $95 \pm 21$ \\
\hline R608W (4) & mSCP2 & $0.61 \pm 0.06$ & -71.7 & -31.8 & -39.9 & $179 \pm 19$ \\
\hline D624W (4) & mSCP2 & $0.41 \pm 0.08$ & -55.6 & -12.2 & -43.4 & $45 \pm 8$ \\
\hline Wild-type (2) & PGNAKL & $0.94 \pm 0.02$ & -42.5 & -5.6 & -36.9 & $547 \pm 6$ \\
\hline R608W (2) & PGNAKL & $0.81 \pm 0.01$ & -51.6 & -15.1 & -36.5 & $635 \pm 25$ \\
\hline D624W (2) & PGNAKL & $0.49 \pm 0.01$ & -54.7 & -18.6 & -36.1 & $763 \pm 0$ \\
\hline
\end{tabular}

${ }^{a}$ Numbers in brackets indicate the number of measurements.

$1.0 \%$, in good agreement with the $60.2-64.4 \%$ of residues in $\alpha$-helices (as a proportion the 329 residue fragment consistently used in crystallisation experiments) found within the various chains of apo-human $\operatorname{Pex} 5 \mathrm{p}(\mathrm{C})$ in PDB entries 2C0M [4] and 2J9Q [5]. However, D624W carries only $48.7 \pm 1.5 \% \alpha$-helix (with a concomitant increase in irregular structure), suggesting that the D624W mutation may alter the folding properties of Pex5p $(\mathrm{C})$. These data show that a specific cargo recognition defect can be seen with the Pex5p R608W mutant when binding full-length SCP2 and that structural impairment was not the cause of this binding defect.

To further clarify the impaired binding between Pex5p (C) mutants and SCP2, we examined the interaction kinetics using the Octet RED96 assay system (Table 4) [6]. Once again, in the absence of the PTS1, no binding could be measured - regardless of whether the presequence in SCP2 or mutations in $\operatorname{Pex} 5 \mathrm{p}(\mathrm{C})$ were present. We detected that relative to wild-type $\operatorname{Pex} 5 \mathrm{p}(\mathrm{C})$, each mutant displayed a 2.0 - 3.5 fold decreases in association rates $\left(\mathrm{k}_{\text {on }}\right)$. Most strongly impaired in $\mathrm{k}_{\text {on }}$ (a 3.5 fold decrease) was the infantile Refsum disease mutant Pex5p(C)S600W [7], a mutation in the 7C-loop (and therefore distant from the ancillary interface) we have previously found not to bind to $\mathrm{MSCP} 2$ in ITC experiments and to impair import of SCP2 and catalase in vivo [4]. In contrast, all mutants displayed very similar dissociation rates $\left(\mathrm{k}_{\text {off }}\right)$ to wild-type $\operatorname{Pex} 5 \mathrm{p}(\mathrm{C})$, with the exception of D624W, which had a 2.7 fold more rapid $\mathrm{k}_{\text {off }}$ from $\mathrm{mSCP} 2$ compared to wild-type. Thus, in line with our previous data, the ancillary interface residues Arg608 and Asp624 do have roles in receptor recognition and binding but at least in the case of
Arg608, play a lesser role in the overall stability of the Pex $5 \mathrm{p}(\mathrm{C}) / \mathrm{SCP} 2$ complex. The impaired $\mathrm{k}_{\text {on }}$ and $\mathrm{k}_{\text {off }}$ demonstrated for D624W presumably result from the structural deviation shown above.

\section{Peroxisomal import of SCP2 in fibroblast complementation assays}

We have complemented Pex5p impaired fibroblast cultures from Zellweger syndrome patient PBD005 [4] with the long isoform of human Pex5p (Pex5p(L)) and Pex5p (L) mutated at residues Arg608 and Asp624. 12, 24 and 48 hours after transfection, the ability of these complemented cells to import the reporter molecule GFPmSCP2 into peroxisomes was assayed by immunofluorescence microscopy (Figure 5). We also determined the localisation of Pex5p(L) using immunofluorescence. The cytosolic distribution of Pex $5 \mathrm{p}(\mathrm{L})$ was not altered by the mutations and all ancillary interface mutants gave rise to a similar punctuate pattern for GFP-mSCP2, characteristic of peroxisomal localisation (Figure 5). Even when the transfected cells were incubated at $40^{\circ} \mathrm{C}$ instead of $37^{\circ} \mathrm{C}$ (to challenge the cells with a stress condition) the Pex5p mutants retained their ability to import GFP-SCP2 into peroxisomes (data not shown). In contrast, both negative controls, using Pex5p(L) S600W and wild-type Pex5p(L) with GFP-mSCP2 $\triangle \mathrm{AKL}$, showed diffuse GFP fluorescence (Figure 5), indicating that in each case GFP-SCP2 is retained in the cytosol. Thus, despite the moderate impairment of in vitro binding caused by mutation at the ancillary interface, the same mutations do not impair $\operatorname{Pex} 5 \mathrm{p}(\mathrm{L})$ mediated peroxisomal import, at least of SCP2 as an import substrate - although subtle alterations in import kinetics cannot entirely be excluded by our

Table 2 Summary of static light scattering data

\begin{tabular}{lccc}
\hline & Pex5p(C) WT & Pex5p(C) R608W & Pex5p(C) D624W \\
\hline Molecular weight $(\mathbf{M w})$ & $32.2^{\mathrm{a}}( \pm 8 \%)$ & $35.6( \pm 25 \%)$ & $30.3( \pm 18 \%)$ \\
\hline Number weighted mean $(\mathbf{M n})$ & $31.0( \pm 8 \%)$ & $34.4( \pm 20 \%)$ & $29.9( \pm 16 \%)$ \\
\hline Polydispersity $(\mathbf{M w} / \mathbf{M n})$ & $1.0( \pm 11 \%)$ & $1.0( \pm 32 \%)$ & $1.0( \pm 25 \%)$ \\
\hline Theoretical $\mathbf{M w}$ & 35.2 & 35.2 & 35.3 \\
\hline
\end{tabular}

${ }^{\mathrm{a}}$ Values are expressed in $\mathrm{kDa}$. 


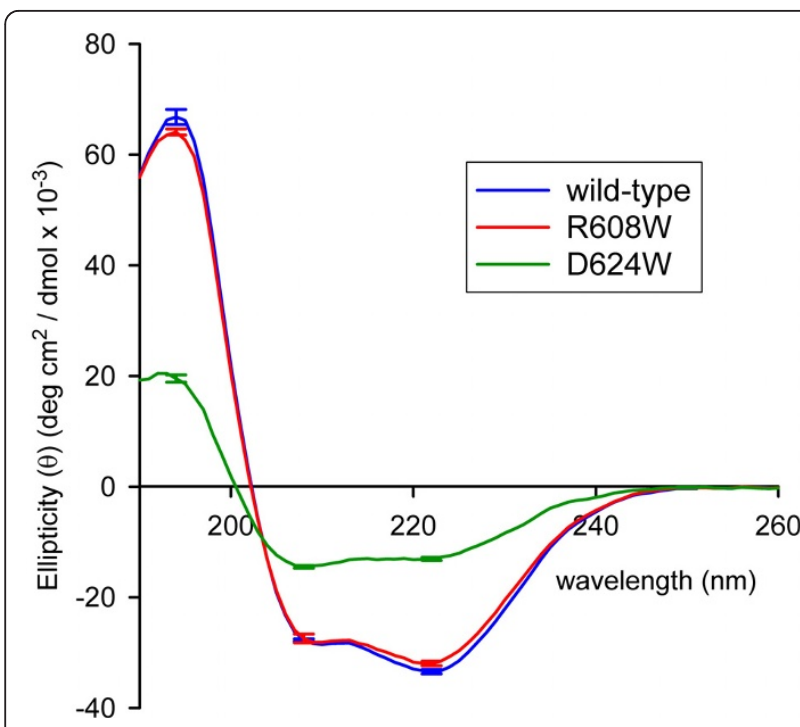

Figure 4 Circular dichroism analysis of wild-type and mutant Pex5p(C). CD spectra of wild-type and the mutant (R608W and D624W) forms of Pex 5 (C). While wild-type and R608W show very similar secondary structure content, D624W does not. See text for details.

localisation assay. However, the presence of the PTS1 and retention of the capacity of $\operatorname{Pex} 5 \mathrm{p}(\mathrm{L})$ to act as a PTS1 binding receptor are essential.

\section{Discussion}

Our data confirm previous findings [4] that the PTS1 of SCP2 is essential for the interaction with the PTS1 receptor Pex5p and for its Pex5p-mediated targeting to the peroxisomal matrix and we additionally characterise the role of the secondary binding site in cargo binding. While mutation of Arg608 in Pex5p(C) impairs the binding affinity, stoichiometry (from two hybrid and ITC experiments) and association rate (from Octet RED96 experiments) with SCP2, its' introduction in vivo does not impair the capacity of Pex $5 \mathrm{p}(\mathrm{L})$ to import SCP2 into peroxisomes, suggesting that the ancillary interface, formed between SCP2 and Pex $5 \mathrm{p}$ plays only a limited role in the import of SCP2.

The R608W and D624W mutations in Pex5p(C), while not causing an obvious phenotype in vivo, do cause some interesting and unexpected behaviour in vitro.

Table 3 Summary of secondary structure estimations from CD spectropolarimetry

\begin{tabular}{lcccc}
\hline Pex5p(C) sample & $\% \boldsymbol{\alpha}$-helix & \% $\beta$-strand & $\%$ other & NRMSD $^{c}$ \\
\hline Wild-type & $60.3 \pm 1.5^{b}$ & $7.0 \pm 1.0$ & $32.6 \pm 0.6$ & $0.012 \pm 0.002$ \\
\hline R608W & $57.0 \pm 1.0$ & $9.0 \pm 1.0$ & $34.0 \pm 0.6$ & $0.011 \pm 0.002$ \\
\hline D624W & $48.7 \pm 1.5$ & $9.0 \pm 1.0$ & $42.3 \pm 0.6$ & $0.017 \pm 0.002$ \\
\hline
\end{tabular}

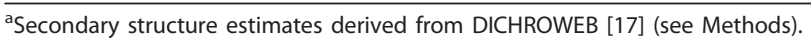

${ }^{\mathrm{b}}$ Mean and standard deviation over three measurements are given.

${ }^{\mathrm{c}}$ Normalised root mean square deviation calculated in DICHROWEB [17].
Firstly, the apparent increase in binding affinity caused by the D624W mutation, an effect that seems to contradict the reduction observed with the R608W mutant. Our CD data indicate that the D624W mutant has altered folding properties, in relation to the wild type and R608W mutant, which may affect the binding to SCP2 in an unexpected way. A reduction in binding affinity can readily be justified, in terms of a loss of contact sites in Pex $5 \mathrm{p}(\mathrm{C})$. However, the reductions in binding stoichiometries of the mutants are less easily explained. We cannot absolutely rule out the possibility that the Pex $5 p$ mutants are able to oligomerise, resulting in a change in the stoichiometry of the interaction. However, our SLS data indicate that both mutants, like the wild type protein, are monomeric in solution, which makes this explanation unlikely. From the Octet RED96 experiments, we see that both mutants exhibit a slower association rate. This, together with the faster dissociation rate observed with the D624W mutant, combined with apparent changes in its' folding properties, could contribute to differences in the stoichiometries.

We are then left with the question as to what the possible function(s) of the secondary interface in PTS1 protein import may be. The capacity for Pex5p to import folded proteins is well known and is likely to represent an essential part of its task since to date no chaperones have been identified within the peroxisomal matrix that could aid in the folding of imported substrates. Therefore, two possible roles for the ancillary interface could be envisaged; increasing the overall stability of the receptor-cargo complex by providing extra contact sites between Pex5p and the PTS1 protein and/or a "quality control" step, allowing Pex5p to check PTS1 proteins for correct folding before importing them into the matrix, since the region in SCP2 recognised by the ancillary interface, unlike the PTS1, is fully folded. However, it may be expected that impairment of either process would result in an inhibition of the import process, which is not the case in our hands. Our data do not rule out the possibility that Pex5p uses this secondary interface with other cargo proteins and indeed, the apparent conservation of the Arg608 residue suggests a potential role in the function of Pex5p. In addition, recent results indicate that other PTS1-containing proteins interact with this same region of Pex5p in a similar way (K. Fodor, personal communication). Therefore, factors additional to the ancillary interface may determine the contribution of this binding site to cargo recognition. Recent data indicate that human PTS1 sequences show a range of binding affinities to Pex $5 p$ [8]. It is not hard to imagine that proteins with a relatively low binding affinity may require additional contact sites, to improve their targeting, while those with a high binding affinity (such as SCP2) would not. The targeting 
Table 4 Summary of binding kinetics measured using Octet RED96

\begin{tabular}{llrr}
\hline Immobilised biotinylated Pex5p(C) & Ligand & Association rate, $\mathbf{k}_{\text {on }}(\mathbf{1 / M s})$ & Dissociation rate, $\mathbf{k}_{\text {off }}(\mathbf{1 / s})$ \\
\hline Wild-type & mSCP2 & $2.3 \times 10^{4}\left( \pm 2.0 \times 10^{2}\right)$ & $5.5 \times 10^{-5}\left( \pm 2.0 \times 10^{-6}\right)$ \\
\hline Wild-type & preSCP2 & $3.5 \times 10^{4}\left( \pm 2.8 \times 10^{2}\right)$ & $7.9 \times 10^{-5}\left( \pm 1.5 \times 10^{-6}\right)$ \\
\hline S600W & mSCP2 & $6.5 \times 10^{3}\left( \pm 3.9 \times 10^{1}\right)$ & $6.0 \times 10^{-5}\left( \pm 1.7 \times 10^{-6}\right)$ \\
\hline S600W & preSCP2 & $7.3 \times 10^{3}\left( \pm 4.8 \times 10^{1}\right)$ & $5.1 \times 10^{-5}\left( \pm 1.8 \times 10^{-6}\right)$ \\
\hline R608W & mSCP2 & $7.2 \times 10^{3}\left( \pm 4.8 \times 10^{1}\right)$ & $7.3 \times 10^{-5}\left( \pm 1.8 \times 10^{-6}\right)$ \\
\hline R608W & preSCP2 & $9.0 \times 10^{3}\left( \pm 6.4 \times 10^{1}\right)$ & $5.0 \times 10^{-5}\left( \pm 1.9 \times 10^{-6}\right)$ \\
\hline D624W & mSCP2 & $1.1 \times 10^{4}\left( \pm 8.3 \times 10^{1}\right)$ & $1.5 \times 10^{-4}\left( \pm 2.3 \times 10^{-6}\right)$ \\
\hline D624W & preSCP2 & $1.3 \times 10^{4}\left( \pm 1.1 \times 10^{2}\right)$ & $8.6 \times 10^{-5}\left( \pm 2.2 \times 10^{-6}\right)$ \\
\hline
\end{tabular}

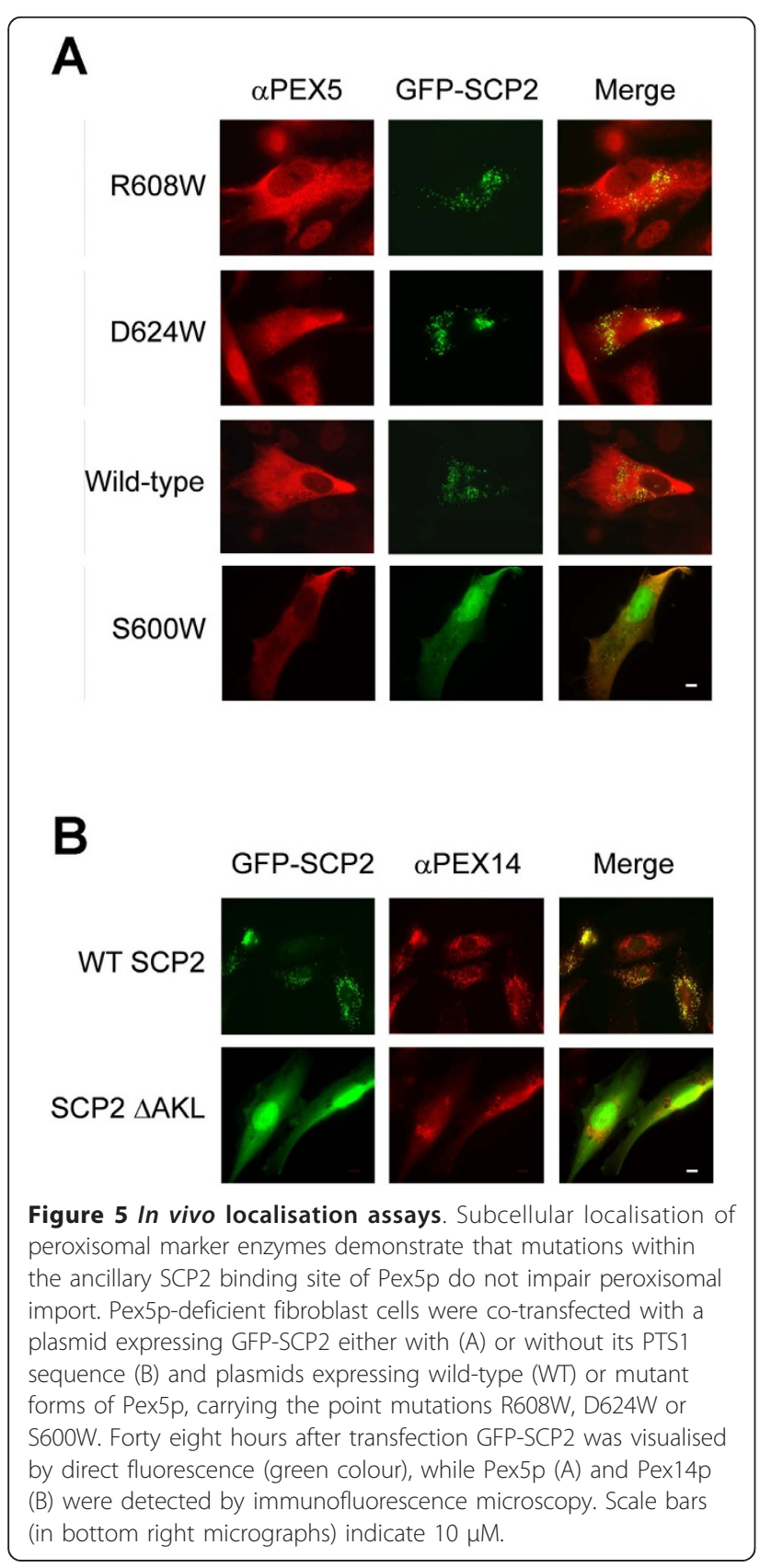

of GFP-SKL to peroxisomes falls into the last category. GFP (being a non-native import substrate commonly used as a reporter) is not expected to interact with the secondary interface in Pex5p, yet it is still efficiently targeted, indicating that SKL is sufficient to allow targeting in the absence of a secondary interface. Consequently, further data on other PTS1 cargo proteins are required, to provide insights into the role of the ancillary interface in peroxisome translocation.

\section{Conclusion}

The data presented in this study clarify that the mechanism of SCP2 sorting to the peroxisome is absolutely PTS1 dependent but independent of the ancillary interface. It remains to be seen if a broad set of proteins destined for the peroxisome lumen utilise this ancillary binding site, or if the majority rely on a more rugged "PTS1 or nothing" selection, as appears to be the case here.

\section{Methods \\ Materials}

Unless otherwise stated, all chemicals were obtained at the highest available purity from Sigma-Aldrich. Restriction enzymes were purchased from New England Biolabs (Ipswich, MA, USA).

\section{Plasmids and cloning}

E. coli expression vectors for the production of human Pex5p $(C)$ (spanning residues 315-639), preSCP2 (1-143) and mSCP2 (21-143) complete with N-terminal $\mathrm{His}_{6}$ GST fusion, cleavable with tobacco etch virus (TEV) protease have been described previously $[4,9]$. Primers described in [9] were also used to facilitate insertion of the four SCP2 variants (preSCP2 (1-143), mSCP2 (21-143), preSCP2 $\triangle \mathrm{AKL}$ (1-140) and $\mathrm{mSCP} 2 \Delta \mathrm{AKL}$ (21-140)) into pPC97 [10] between SalI and NotI, to generate fusions with Gal4 DNA binding domain (Gal4DB) for yeast two-hybrid analysis. The fusion of $\mathrm{HsPex} 5 \mathrm{p}(\mathrm{C})$ and the Gal4 activation domain (Gal4AD) was made by cloning the NcoI-NotI fragment from the 
HsPex5p(C) E. coli expression vector into pPC97. Point mutations were introduced into the various Pex5p constructs using the QuikChange ${ }^{\mathrm{TM}}$ site directed mutagenesis kit (Stratagene) using either the Gal4AD Pex5p(C) plasmid, the $\mathrm{His}_{6}-\mathrm{GST} \operatorname{Pex} 5 \mathrm{p}(\mathrm{C})$ plasmid, or the pcDNA3 derived expression vector pGD106 [11] as templates. Details of the primers used can be seen in Table 5. The Pex5p S600W mutant has been described previously [4]. All constructs were confirmed by DNA sequencing.

\section{Strains and culture conditions}

Recombinant production of human $\operatorname{Pex} 5 \mathrm{p}(\mathrm{C})$, preSCP2 and $\mathrm{mSCP} 2$ in $E$. coli has been described before $[4,9]$. The yeast strain S. cerevisiae PCY2 (MATA, $\Delta$ gal4, Igal80, URA3::GAL1-lacZ, lys2-801, his3-4200, trp1$\triangle 63$, leu2, ade2-101) was used for two-hybrid analysis. Yeast transformations were performed as described in [12]. Transformants were grown on minimal medium containing $0.67 \%$ yeast nitrogen base (Difco), $2 \%$ glucose and amino acids $(20 \mu \mathrm{g} / \mathrm{ml})$ as required.

\section{Yeast two-hybrid analysis and Western blotting}

GAL4 based yeast two hybrid analysis was conducted as described in [10] with $\beta$-galactosidase enzyme activity determination being performed as in [13]. Samples of lysates taken from the $\beta$-galactosidase assay were analysed by Western blotting for human Pex5p expression levels. Proteins were separated on a SDS-polyacrylamide gel and blotted onto a nitrocellulose membrane using a semi-dry system. Antibodies used were directed against S. cerevisiae hexokinase (generous gift of $\mathrm{H}$. van der Spek, FNWI, Amsterdam, The Netherlands) and human Pex5p described in [14] Antibody binding was detected with secondary antibodies labeled with IRDye 680 infrared dye on an Odyssey imager (LI-COR Biosciences).

\section{Protein preparation and biotinylation}

Preparation of Pex5p $(\mathrm{C})$, preSCP2 and $\mathrm{mSCP} 2$ has been described previously [4,9]. Mutant $\operatorname{Pex} 5 \mathrm{p}(\mathrm{C})$ proteins were prepared in a similar way as described in [4]. Purity was monitored by SDS-PAGE and mass spectrometry. For concentration determination, proteins were denatured in $8 \mathrm{M}$ urea and the $\mathrm{A}_{280 \mathrm{~nm}}$ was measured.

Table 5 PCR primers used in this study

\begin{tabular}{ll}
\hline Primer name & $\mathbf{5}^{\prime}$-3' sequence \\
\hline HsPex5p: & CATCTGGAGCACCCTGTGGTTGGCATTGTCTATGTTAGG \\
R608Wf & \\
HsPex5p: & CCTAACATAGACAATGCCAACCACAGGGTGCTCCAGATG \\
R608Wr & \\
HsPex5p: & CCTATGGGGCAGCCTGGGCGCGGGATCTGTC \\
D624Wf & \\
HsPex5p: & GACAGATCCCGCGCCCAGGCTGCCCCATAGG \\
D624Wr & \\
\hline
\end{tabular}

Extinction coefficients were calculated using the method of Gill and von Hippel [15]. $\operatorname{Pex} 5 \mathrm{p}(\mathrm{C})$ wild-type and mutants were biotinylated using the EZ-Link ${ }^{\circledR}$ SulfoNHS-Biotinylation Kit (Pierce) as described by the manufacturer. Using the 4'-hydroxyazobenzene-2-carboxylic acid/avidin method, between 1 and 2 moles of biotin were found incorporated with 1 mole of $\operatorname{Pex} 5 \mathrm{p}(\mathrm{C})$.

\section{Isothermal titration microcalorimetry (ITC)}

ITC was performed as described in [4]. Measurements were conducted using a MicroCal VP-ITC using either wild-type or mutant (R608W or D624W) forms of Pex5p (C) titrated with either $\mathrm{mSCP} 2$ or a peptide derived from the C-terminus of SCP2 [4]. The peptide, PGNAKL, was synthesised by Sigma-Aldrich at $>95 \%$ purity.

\section{Circular dichroism spectropolarimetry}

Circular dichroism spectropolarimetry (CD) was performed as described in [16] with $\operatorname{Pex} 5 \mathrm{p}(\mathrm{C})$ at a concentration of $2-5 \mu \mathrm{M}$ in $10 \mathrm{mM}$ Potassium Phosphate buffer $\mathrm{pH}$ 7.4. Each spectrum presented is the average of three measurements. Standard deviations in ellipticity are shown with error bars. Spectra were background subtracted and helical content estimated via the DICHROWEB interface [17], using CDSSTR [18] with the SP175 basis set of spectra.

\section{Static light scattering}

The procedure used for static light scattering (SLS) analysis is described in [19].

\section{Octet RED96 kinetic assays}

To assess the binding kinetics of $\operatorname{Pex} 5 \mathrm{p}(\mathrm{C})$ wild-type and point mutants to the four SCP2 variants, the Octet RED96 instrument (ForteBio) was used [6]. All steps were carried out in phosphate-buffered saline (PBS) at a rotation rate of $1000 \mathrm{rpm}$. Streptavidin conjugated sensors were hydrated for 10 minutes prior to use. The biotinylated sample proteins were immobilised onto the sensor at a concentration of $20 \mu \mathrm{g} / \mathrm{mL}$. The experiment proceeded as follows; baseline - 60 seconds, biotinylated-sample loading - 300 seconds, baseline 2 - 60 seconds, association - 1800 seconds and dissociation - 1800 seconds. Data were acquired and assessed using the custom ForteBio software Data Acquisition v6.2 and Data Analysis v6.3. The data were processed with the Savitzky-Golay filter prior to analysis. Lines of best fit were generated locally based on a 1:1 model to determine the relative binding kinetics.

\section{In vivo peroxisome import assays}

Culturing and transfection of the Pex5p deficient human skin fibroblast cells for Zellweger syndrome patient PBD005 was performed as described in [4]. 12, 24 and 
48 hours after transfection, cells were fixed onto cover glasses with 3\% formaldehyde in PBS, permeablised with $1 \%$ Triton X-100 in PBS and subjected to immunofluorescence microscopy. To test for temperature-sensitive import defects, the temperature of cell culture was shifted from 37 to $40^{\circ} \mathrm{C}$ for 2 days. Polyclonal rabbit antibodies directed against human Pex14p are described in [20]. Polyclonal rabbit anti-Pex5p antibodies were raised against recombinant $\mathrm{His}_{6}$-tagged Pex5p expressed in E. coli and purified as described previously [14]. Secondary antibodies were conjugated with Alexa Fluor 594 (Invitrogen). Samples were also inspected for GFP fluorescence. All micrographs were recorded on a Zeiss Axioplan 2 microscope with a Zeiss Plan-Apochromat 63x/1.4 oil objective and an Axiocam MR digital camera and were processed with AxioVision 4.2 software (Zeiss).

\footnotetext{
Abbreviations

CD: Circular dichroism spectropolarimetry; GFP: green fluorescent protein; ITC: isothermal titration calorimetry; PBS: phosphate buffered saline; PTS: peroxisomal targeting signal; SCP2: sterol carrier protein 2; SLS: Static light scattering; TPR: tetratricopeptide repeat.
}

\section{Acknowledgements}

The authors would like to thank Krisztian Fodor for sharing unpublished results, Lise Hafkenscheid \& Matt Groves for technical assistance and other members of our labs for stimulating discussions. This work was partly funded by a grant from the Academic Medical Center awarded to B.D., a Rubicon Fellowship (825.08.023) from the Netherlands Organisation for Scientific Research (NWO) awarded to C.P.W and by 3D-REPERTOIRE (EC, LSHG-CT-2005512028), grant awarded to M.W.

\section{Author details \\ ${ }^{1}$ EMBL-Hamburg, c/o DESY, Notkestraße 85, 22603 Hamburg, Germany. ${ }^{2}$ Department of Medical Biochemistry, Academic Medical Center, University of Amsterdam, Meibergdreef 15, 1105 AZ Amsterdam, The Netherlands. ${ }^{3}$ Phylogica, 100 Roberts Road, Subiaco, 6008 WA, Australia. ${ }^{4}$ Department of Systems Biochemistry, Institute for Physiological Chemistry, Faculty of Medicine, Ruhr University of Bochum, 44780 Bochum, Germany. ${ }^{5}$ School of Biomedical, Biomolecular and Chemical Sciences, MCS Building (M310), University of Western Australia, 35 Stirling Highway, Crawley, 6009 WA, Australia. ${ }^{6}$ ARC CoE in Plant Energy Biology, MCS Building (M316), University of Western Australia, 35 Stirling Highway, Crawley, 6009 WA, Australia.}

\section{Authors' contributions}

Experimental data were collected and analysed by CPW, NS, CAT, MvdB, SDVH, CSB, WS and WAS. The study was conceived and designed by CPW, RE, WS, MW and WAS. CPW, NS, CAT, RE, CSB, BD, WS, MW and WAS interpreted the data and prepared the manuscript. All authors read and approved the final manuscript.

Received: 9 August 2010 Accepted: 4 March 2011 Published: 4 March 2011

\section{References}

1. Girzalsky W, Platta HW, Erdmann R: Protein transport across the peroxisomal membrane. Biol Chem 2009, 390(8):745-751.

2. Lanyon-Hogg T, Warriner SL, Baker A: Getting a camel through the eye of a needle: the import of folded proteins by peroxisomes. Biol Cell 2010, 102(4):245-263.

3. Gatto GJ Jr, Geisbrecht BV, Gould SJ, Berg JM: Peroxisomal targeting signal-1 recognition by the TPR domains of human PEX5. Nat Struct Biol 2000, 7(12):1091-1095.

4. Stanley WA, Filipp FV, Kursula P, Schuller N, Erdmann R, Schliebs W, Sattler M, Wilmanns M: Recognition of a functional peroxisome type 1 target by the dynamic import receptor pex5p. Mol Cell 2006, 24(5):653-663.

5. Stanley WA, Pursiainen NV, Garman EF, Juffer AH, Wilmanns M, Kursula P: A previously unobserved conformation for the human Pex $5 p$ receptor suggests roles for intrinsic flexibility and rigid domain motions in ligand binding. BMC Struct Biol 2007, 7:24.

6. Cooper MA: Optical biosensors: where next and how soon? Drug Discov Today 2006, 11(23-24):1061-1067.

7. Shimozawa N, Zhang Z, Suzuki Y, Imamura A, Tsukamoto T, Osumi T, Fujiki Y, Orii T, Barth PG, Wanders RJA, et al: Functional heterogeneity of C-terminal peroxisome targeting signal 1 in PEX5-defective patients. Biochemical and Biophysical Research Communications 1999, 262(2):504-508.

8. Ghosh D, Berg JM: A proteome-wide perspective on peroxisome targeting signal 1(PTS1)-Pex5p affinities. J Am Chem Soc 132(11):3973-3979.

9. Stanley WA, Versluis K, Schultz C, Heck AJ, Wilmanns M: Investigation of the ligand spectrum of human sterol carrier protein 2 using a direct mass spectrometry assay. Arch Biochem Biophys 2007, 461(1):50-58.

10. Chevray PM, Nathans D: Protein interaction cloning in yeast: identification of mammalian proteins that react with the leucine zipper of Jun. Proc Natl Acad Sci USA 1992, 89(13):5789-5793.

11. Braverman N, Dodt G, Gould SJ, Valle D: An isoform of pex $5 p$, the human PTS1 receptor, is required for the import of PTS2 proteins into peroxisomes. Hum Mol Genet 1998, 7(8):1195-1205.

12. Van der Leij I, Franse MM, Elgersma Y, Distel B, Tabak HF: PAS10 is a tetratricopeptide-repeat protein that is essential for the import of most matrix proteins into peroxisomes of Saccharomyces cerevisiae. Proc Natl Acad Sci USA 1993, 90(24):11782-11786.

13. Klein AT, Barnett P, Bottger G, Konings D, Tabak HF, Distel B: Recognition of peroxisomal targeting signal type 1 by the import receptor Pex $5 p$. J Biol Chem 2001, 276(18):15034-15041.

14. Schliebs W, Saidowsky J, Agianian B, Dodt G, Herberg FW, Kunau WH: Recombinant human peroxisomal targeting signal receptor PEX5. Structural basis for interaction of PEX5 with PEX14. J Biol Chem 1999, 274(9):5666-5673.

15. Gill SC, von Hippel PH: Calculation of protein extinction coefficients from amino acid sequence data. Anal Biochem 1989, 182(2):319-326.

16. Opalinski L, Kiel JA, Williams C, Veenhuis M, van der Klei IJ: Membrane curvature during peroxisome fission requires Pex11. Embo J 2011, 30(1):5-16.

17. Whitmore L, Wallace BA: Protein secondary structure analyses from circular dichroism spectroscopy: methods and reference databases. Biopolymers 2008, 89(5):392-400.

18. Sreerama N, Woody RW: Estimation of protein secondary structure from circular dichroism spectra: comparison of CONTIN, SELCON, and CDSSTR methods with an expanded reference set. Anal Biochem 2000, 287(2):252-260.

19. Nettleship JE, Brown J, Groves MR, Geerlof A: Methods for protein characterization by mass spectrometry, thermal shift (ThermoFluor) assay, and multiangle or static light scattering. Methods Mol Biol 2008, 426:299-318.

20. Will GK, Soukupova M, Hong X, Erdmann KS, Kiel JA, Dodt G, Kunau WH, Erdmann R: Identification and characterization of the human orthologue of yeast Pex14p. Mol Cell Biol 1999, 19(3):2265-2277.

21. Thompson JD, Higgins DG, Gibson TJ: CLUSTAL W: improving the sensitivity of progressive multiple sequence alignment through sequence weighting, position-specific gap penalties and weight matrix choice. Nucleic Acids Res 1994, 22(22):4673-4680.

22. Bond CS, Schuttelkopf AW: ALINE: a WYSIWYG protein-sequence alignment editor for publication-quality alignments. Acta Crystallogr D Biol Crystallogr 2009, 65(Pt 5):510-512.

23. Kabsch W, Sander C: Dictionary of Protein Secondary Structure - PatternRecognition of Hydrogen-Bonded and Geometrical Features. Biopolymers 1983, 22(12):2577-2637.

doi:10.1186/1471-2091-12-12

Cite this article as: Williams et al:: The Peroxisomal Targeting Signal 1 in sterol carrier protein 2 is autonomous and essential for receptor recognition. BMC Biochemistry 2011 12:12. 